\title{
Character Matching and the Locke Pocket of Belief
}

\begin{abstract}
The Lockean thesis of full belief maintains that an individual believes a proposition just when he has a high enough level of confidence. The trouble with the Lockean thesis, according to probabilists like Richard Jeffrey, is that it licenses throwing away perfectly good information. Some proponents of the Lockean thesis—call them Harmonious Lockeans - maintain that reconciling qualitative and quantitative belief will need to occur on Jeffrey's terms. To this end, Scott Sturgeon has advanced a normative principle for Harmonious Lockeanism called Character Matching, which this essay criticizes through means of a counterexample and, with the help of a model of Henry Kyburg's, an alternative model for Harmonious Lockean belief that turns the Principle of Character Matching inside out. The upshot is that the character of evidence provides no corresponding normative constraint on the character of belief. A further consequence is that the disposition to fully believe, while dependent on a subject's practical interests, does not depend upon the total magnitude of the stake put at risk.
\end{abstract}

\section{INTRODUCTION}

The Lockean thesis maintains that an individual fully believes a proposition $\varphi$ just when he has a high level of confidence in $\varphi$. The trouble with the Lockean thesis, according to probabilists like Richard Jeffrey, is that it licenses throwing away perfectly good information. Any numerically determinate degree of belief that happens to fall above the Lockean's threshold for full acceptance is abandoned in favor of a qualitative label, 'full belief'. What orthodox probabilists want to know is whether there is anything gained in the exchange.

To be sure, some probabilists take the case against full belief too far: the idea that full belief can be entirely replaced by credal probability, for example, is a nonstarter. Probability presupposes possibility, and judgments of possibility-even if only to pick this algebra rather than that one for your probability structure-are categorical. Further still, there are alternatives to probabilism. Henry Kyburg's lottery paradox (Kyburg 1961) was a campaign button for his $\varepsilon$-acceptance theory of evidential probability, which is deeply at odds with probabilism. David Makinson's puzzle about prefaces (Makinson 1965) preceded his important work on systems that facilitate genuine inductive expansion (Makinson 2005), yet inductive expansion is notoriously difficult to square with probabilism (Levi 1980; Paris and Simmonds 2009).

Nevertheless, Kyburg's lottery paradox and Makinson's paradox of the preface have developed a life of their own as Rorschach tests for the metaphysics of rational belief. In what sense, if any, is belief categorical? It what sense, if any, is it gradable? 
How weird does the logic have to be to have it both ways? The orthodox probabilist answers none, completely, and too weird. The conventional Lockean says that rational full belief summarizes information conveyed by a high degree of credal probability and the logic for full belief is classical except that it will not be closed under conjunction. Here then is a threshold acceptance view that is purported to be in harmony with the spirit of probabilism: 'Full belief' is simply code for 'has high credal probability'. But then the harmonous Lockean faces the question that started us off: why bother?

\section{Character Matching}

In the course of defending a mission for harmonious Lockeanism, Scott Sturgeon introduces a normative principle called character matching which maintains that the character of a belief should match the character of the evidence upon which it is based.

When evidence is essentially sharp, it warrants a sharp or exact attitude; when evidence is essentially fuzzy ... it warrants at best a fuzzy attitude. In a phrase: evidential precision begets attitudinal precision; and evidential imprecision begets attitudinal imprecision (Sturgeon 2008, p. 159).

Sturgeon's idea is that everyday evidence is often imprecise in character; so, by the principle of character matching, everyday evidence will seldom rationalize numerically determinate credences but instead will typically rationalize only regions of credal space. Yet, when imprecise evidence for a claim occupies the range $[\theta, 1]$, for a suitably high threshold value $\theta$ between $1 / 2$ and 1 , Sturgeon claims that 'lending that confidence to [the] claim functions exactly like believing it in a threshold-based way' (2008, p.160). Harmonious Lockeanism is thus conceived to be an extension of probabilism to cover the majority of cases in which everyday evidence is strong but imprecise. So, the harmonious Lockean is not throwing away information when he fully believes: he is making the best of the information he's got.

That commonplace evidence is more often precise than imprecise is surely right. I may observe that $A$ is more frequent than $B$ either by rough observation of the occurrence of $A$ 's versus the occurrence of $B$ 's or by more careful methods of statistical estimation. Neither case yields numerically determinate frequencies, however, unless of course my estimation is gotten directly from a complete description of the occurrence of $A$ 's and the occurrence of $B$ 's in the population. If I know the proportion of red balls in an urn is exactly 0.85 because I loaded the balls into the urn myself, say, then I know that a sample set of 100 balls from the urn should contain roughly 85 red balls. I can leverage my knowledge of the population, i.e., the urn, to calibrate my sampling mechanism. If instead I do not know the proportion of red balls in the urn but I draw several 100-ball sample sets randomly, never observe less than 80 red balls in a sample and likewise never observe more than 90 balls in a sample, then I may estimate (conservatively) that the proportion of red balls in the urn is between 0.8 and 0.9 . In this manner I learn about the proportion of red balls in the population by observing samples from that population. Why? Because it is extremely unlikely to go through an exercise like this one, observe the same proportions of red balls from a set of 100-ball 
samples, yet have it be the case that I am drawing from an urn whose proportion of red balls is in fact outside of the interval $[0.8,0.9]$. We may fully believe that the actual proportion of red balls is between 0.8 and 0.9 because, by manipulating the number of samples drawn, we may control the risk faced from adopting this attitude in error. We may judge that the proportion of red balls in the urn is within [0.8, 09], or that the proportion of red balls is roughly [0.8, 0.9]. If we happen to know that error in our samples is distributed normally, we may judge that the proportion of red balls is roughly 0.85 . Analogously, we may judge that the length of a table leg is $71 \pm .03$ $\mathrm{cm}$, or that it is roughly $71 \mathrm{~cm}$, or roughly between 70.97 and 71.03 units centimeter in length-depending on what is known about the instrument, the measurement procedure, and the conditions under which the measurements are taken.

But while it is clear that much of our evidence is imprecise, it is far less clear what bearing this should have on rational full belief. Sturgeon maintains that high imprecise credence is identical to full belief, and the linchpin to his argument is the normative principle that 'epistemic perfection demands character match between evidence and attitude' (2008, p. 160).

One might probe whether the character matching principle is true by considering cases where precise evidence appears to at best warrant only imprecise belief. Imagine that Fen the fence is trying to sell You $^{1}$ a rigged lottery ball machine. Fen tells You that the machines is calibrated to dispense a red ball $70 \%$ of the time and to dispense an even numbered ball $60 \%$ of the time, and that is all that You are told about the machine. A ball is dispensed. Arguably, before observing the ball, Your credence that it is red is 0.7 and Your credence that the ball is even is 0.6. However, Your credence that the ball is both red and even is indeterminate, taking any value between 0.3 and 0.7. ${ }^{2}$ Here the character of Your evidence is precise but incomplete: knowing the probability that a dispensed ball is red and the probability that a dispensed ball is even warrants at best an imprecise attitude about the ball being both red and even.

The example is not a problem for Sturgeon, however. The reason that Your disposition toward the proposition [the ball is both red and even] is a credal state bound by the closed interval $[0.3,0.7]$ rather than a numerically precise credal probability is due directly to Your evidence, which is that the probability of the ball being both red and even is between 0.3 and 0.7 . Thus, rather than being an objection to the character matching principle, the example instead appears to be a sterling example: Your imprecise belief that the ball is both even and red matches the imprecise character of Your available evidence.

Although the character of evidence and attitude appear to match in this example they do not match as a rule. To illustrate why, imagine that it is the middle of the night and Claudius cannot sleep because of a recurring cough. Groggy, he makes his way in

\footnotetext{
${ }^{1}$ Following a long tradition that includes Walley, de Finetti, and Good, I sometimes use 'You' to denote an intentional system and invite you, the reader, to play along in the role.

${ }^{2}$ In general, for arbitrary propositions $A$ and $B$, if $p(A)$ and $p(B)$ are defined with respect to a probability structure $M$, then with respect to $M$ we have (Wheeler 2006):

- $p(A \wedge B) \in[\max (0, p(A)+p(B)-1), \min (p(A), p(B))]$, and

- $p(A \vee B) \in[\max (p(A), p(B)), \min (p(A)+p(B), 1)]$.
} 
the dark to the medicine cabinet for cough syrup, finds a bottle, unscrews the top, and swallows a liquid that burns rather than soothes his throat. Panicked, he gropes for the light to see what he has ingested, knocking to the floor and shattering two bottles, $A$ and $B$. Claudius determines that he drank from one of the two, neither of which contains cough syrup. Although unable to determine for certain which bottle he drank from, he fully believes that $D$ [it is more likely that Claudius drank from $A$ than from $B]$.

At hospital the attending physician, Ridgeon, tells Claudius that $A$-poisoning is best treated by rest, whereas the recommended treatment for $B$-poisoning is to have ones stomach pumped. Pumping is inadvisable for $A$-poisoning, however, and while sleeping off $B$-poisoning won't kill him, it entails a much longer recovery period than stomach pumping. To make the example concrete, suppose Ridgeon's utilities for these intervention options are given by the following table.

Table 1: Ridgeon's payoff table

\begin{tabular}{l|cc} 
& A & B \\
\hline Rest & 1 & 0.1 \\
Pump & 0 & 0.9
\end{tabular}

Now suppose that Claudius has told Ridgeon the entire story so far except that he has withheld reporting to the doctor that $D$, that it is more likely that he ingested $A$ than $B$. Based on the partial information that Ridgeon has about Claudius's case, Ridgeon's preference about which intervention is best for Claudius is highly indeterminate, ranging from 0.1 to 1 for rest and from 0 to 0.9 for the pump. The doctor's dilemma is so because, at this stage, Ridgeon has no evidence whatsoever about whether it was $A$ or $B$ that Claudius ingested: for Ridgeon, the probability that Claudius drank $A$ is within $[0,1]$ and the probability that Claudius drank $B$ is within $[0,1]$.

Now suppose that Claudius offers up to Ridgeon the missing piece of information. With the addition of $D$, Ridgeon's evidence is that the probability that Claudius has $A$ poisoning is within $(0.5,1]$ and the probability that Claudius has $B$-poisoning is within $[0,0.5]$. The new item of evidence is very imprecise, offering only slight evidence for $A$-poisoning over $B$-poisoning. Indeed, the evidence for $A$-poisoning is well outside the 'bel-region' that Sturgeon imagines is the top 'five to fifteen percent of the scale' for credal probability that is supposed to sustain threshold-based belief (2008, pp. 1601). Yet, although imprecise, notice that this evidence suffices to resolve the doctor's dilemma decisively in favor of rest. ${ }^{3}$ Ridgeon's attitude toward the proposition $R$ [Rest is the best treatment for Claudius] should change from an uncommitted attitude

\footnotetext{
3 To simplify matters, one may calculate expected utility (eu) on the closed interval $[0.5,1]$ instead of the clopen interval $(0.5,1)$ without loss of generality. (See note 4.) There are four expected utilities to calculate: (1) eu(Rest) $=1$, when the probability that $A$ is maximal and $B$ is minimal; (2) eu(Rest) = 0.44 , when the probability of $A$ is minimal and $B$ is maximal; (3) eu(Pump) $=0.45$, when $B$ is maximal, and $(4) \mathrm{eu}($ Pump $)=0$. Because (pump, A) $=0$ in Table 1 , we may ignore those probability values in the last pair of calculations for Pump.
} 
to full rational belief upon receiving the highly imprecise evidence about which bottle Claudius drank from. ${ }^{4}$

Curiously, the doctor's full belief that $R$ is in a sense made stronger rather than weaker by the imprecise character of $D$. Were Ridgeon an orthodox probabilist and insist upon extracting a numerically precise credence from Claudius about his belief that he drank from $A$, his request would add nothing to support Ridgeon's full belief that rest is best, and we might fairly question the doctor's good judgment for bothering to ask.

In short, Ridgeon's full belief that $R$ rests on evidence about $A$-poisoning whose imprecision extends beyond the bel-region to correspond to a high-threshold full belief. What's more, and mentioned as an aside in footnote 4, there is decisive evidence in this case in favor of rest even when the lower-bound for $A$ poisoning is strictly below $1 / 2$. The upshot is that if Claudius either (1) reported that he more likely drank from bottle $A$ than from $B$, (2) reported that is no more than slightly more likely that he ingested $B$ than $A$, or (3) reported any numerically precise credence strictly greater than $0.44 \overline{4}$ that he drank $A$ rather than $B$, then Ridgeon would have formed the same categorical belief that rest is the best treatment for Claudius. None of these three options describes evidence in the bel-region, nor is the region of credal probability associated with $R$ necessarily in the bel-region.

\section{Pocket of Belief}

Full belief and credence do not necessarily match in character nor should they. Credal probability (degrees of belief) encodes a disposition to make a collection of bets on the truth of $\varphi$. (That's Ramsey.) Credal states are a set of admissible credal probability functions, where the conditions for admissibility are a subtle affair. (That's Levi.) The disposition to fully believe $\varphi$, on the other hand, is the disposition to act as if $\varphi$ were true relative to some specified range of actions. This is not the same thing as a disposition to accept bets on $\varphi .^{5}$

We imagine that Ridgeon, once he has the last piece of evidence $(D)$ about Claudius's case, acts as if it is true that rest is the best treatment for him. It is in this respect that full belief is contextually dependent, since an agent's disposition to act as if $\varphi$ is true may vary across contexts even when both the possible set of actions and the evidence for $\varphi$ are held constant.

Some think that this observation about full belief means that the disposition to fully believe depends on the magnitude of the stake. 'The more you care the less you know', as Jason Stanely has put it. ${ }^{6}$ And there are plenty of examples that appear to

\footnotetext{
${ }^{4}$ Notice that a decisive resolution in favor of $R$ does not even depend on the evidence pointing in favor of Claudius having consumed $A$ rather than $B$. The same result holds for all probability functions $p$ such that $p(A)$ is strictly greater than $0.44 \overline{4}$.

5 The probability of a sequence of 50 flips of a fair coin all landing heads is $8.882 \times 10^{-16}$ and ordinarily we act as if this outcome will not occur. But, no one is willing to offer odds of $\$ 1$ to $\$ 1.126$ quadrillion against seeing 50 straight heads; Wall Street traders are a cautionary exception. Furthermore, few would bother to take those odds even if offered.

${ }^{6}$ But the idea goes back at least to R. B. Braithwaite (1946).
} 
support this view. A farmer might act as if a vaccine for flu is non-toxic to his pigs, but refuse to act as if the same vaccine is non-toxic to his children. In short, he may fully believe that the injection is safe for his pigs but not for his kids. This may be so even if the vaccine's fatality rate for children is less than the fatality rate for pigs. The farmer simply values his children much more than he values his pigs, viewing the risk of error acceptable for his pigs but not for his kids.

But focusing on the magnitude of the risk of being wrong is only half of the story; we must also focus on the potential payoff from being right. Return again to our farmer and suppose the question before him now is whether to inoculate his pigs and his kids against a relatively new strain of Escherichia coli which affects $1 / 3$ of each population. The new antibiotic poses identical risk to swine and humans, killing 1 out of 15 in each treated group. However, whereas pigs almost always recover from this strain of E. Coili on their own, children rarely survive it. Faced with this situation, the farmer would give the antibiotic to his children but not to his pigs. That is, in this case the farmer would fully believe that the treatment is non-toxic to his children but toxic to his pigs. Although the farmer values his children much more than he values his pigs, it is still the case that he values his pigs.

What this last example illustrates is that although full belief is context dependent, it does not depend upon the total magnitude of the stake put at risk, as stakes-relative views imagine, but instead depends on the ratio of the amount put at risk $(r)$ by acting as if $\varphi$ were true when $\varphi$ is false, to the amount gained ( $w$ ) by acting as if $\varphi$ were true when $\varphi$ is true. ${ }^{7}$ Adopting a model of Kyburg's, ${ }^{8}$ we say that an agent fully $(r: w)$ believes that $\varphi$ with respect to a context $C$ of available actions iff: for any act $A \in C$, if

1. the agent judges $A$ to cost $r^{*}$ if $\varphi$ is false,

2. the agent judges $A$ to payout $w^{*}$ if $\varphi$ is true,

3. $r^{*} / w^{*}<r / w$, then

the agent acts as if $\varphi$ is true.

If an agent fully $(r: w)$ believes that $\varphi$ (with respect to some $C$ ), then it follows that he fully ( $w: r$ ) disbelieves $\neg \varphi$ in $C$. Since to risk $r$ is to potentially gain $-r$, to gain $w$ is to have risked $-w,-r /-w=w / r$, and $\neg \varphi$ is false if and only if $\varphi$ is true, it follows that full ( $w: r$ ) disbelief that $\neg \varphi$ (in $C$ ) holds just in case, for any act $A \in C$, if (1) the agent judges to cost $w^{*}$ if $\neg \varphi$ is false, (2) to payout $r^{*}$ if $\neg \varphi$ is true, (3) $w^{*} / r^{*}>w / r$ (equivalently, $\left.-r^{*} /-w^{*}<r / w\right)$, then the agent acts as if $\neg \varphi$ is false. An agent $(r: w)$ suspends judgment with respect to $\varphi$ in $C$ if the agent neither fully $(r: w)$ believes $\varphi$ in $C$, nor fully disbelieves $\varphi$ in $C$. From the conjugacy relation between belief and disbelief, i.e., fully $(r: w)$ belief that $\varphi$ in $C$ if and only if full $(w: r)$ disbelief that $\neg \varphi$ in $C$, we say that $r / w$ forms a pocket of belief.

\footnotetext{
${ }^{7}$ We assume that an agent has a cardinal utility function over states of the world such that his utilities are linear and satisfy the standard axioms.

${ }^{8}$ An earlier version of the following risk-reward theory of full belief appears in (Kyburg 1990, pp. 244-55)
} 
To illustrate, suppose that the ratio $1 / 20$ represents the pocket of belief for ordinary contexts. This means that an agent who fully $(1: 20)$ believes the proposition $T$ [lottery ticket \#591 losses] would judge his loss-assume the ticket is his- to be $r^{*}$ if $T$ is false, the payout to be $w^{*}$ if $T$ is true, and the risk-reward ratio $r^{*}: w^{*}$ associated with his acting 'as if $T$ is true' would be strictly less than $1 / 20$. Suppose now that 1000 lottery tickets are sold for $\$ 1.00$ each and the house takes a penny from each ticket sale, leaving $\$ 990$ as the payoff to a single, fairly drawn ticket. And let's suppose a context where the most consequential action the agent considers is whether to discard the ticket before the drawing. Then, the agent 'loses' $r^{*}=-\$ 989$ acting as if $T$ when $T$ is false, and is 'rewarded' $w^{*}=-\$ 1$ if $T$ is true. Yet, $1 / 989 \ngtr 20 / 1$, so the agent does not act as if $\neg T$ is false, and he does not act as if $T$ is true, either. Rather, he will, like most of us would under ordinary circumstances, suspend judgment about $T$, perhaps waiting until the outcome of the lottery is announced to decide what to do with the ticket. Here the character of the evidence for $T$ is in the bel region, but the agent neither fully believes $T$ nor fully disbelieve it: he has a good reason to suspend judgment.

Given the same evidence about $T$, an agent's attitude may change from suspended judgment to full belief either by adjusting the pocket of full belief with respect to $T$, say to $1: 990$, or by creating a genuine risk for acting as if $T$ when $T$ is false. However, it might be difficult to reconcile a 1/990 pocket of belief with the ordinary terms for a lottery drawing, and adjusting the risk-reward ratio to drag $T$ inside the pocket of full belief may be to change the example altogether. No matter. The reluctance to fully believe $T$ in this context provides no sound basis for generalization: there are plenty of so-called 'lottery propositions' (Hawthorne 2004) that are consonant with the pocket of ordinary full belief. For example, return to the length of the table leg mentioned earlier. For the sake of argument, suppose that there is likewise a 1 in 1000 chance that the true length of table leg \#591 is not within the interval estimate $71 \pm .03 \mathrm{~cm}$ that is warranted by the measurement. Unlike lottery tickets, suspending judgment until the announcement of the table leg's true length is not an option. What's more, there are appreciable rewards to fully believing $L$ [Table leg \#591 is $71 \pm .03 \mathrm{~cm}$ ] when $L$ is true: someone's acting as if $L$ were true figured in the table's construction, for instance. So, while there is a non-negative risk to acting as if $L$ is true when it is nota wobbly table, an unhappy customer - the reward in most contexts makes this a risk worth taking. Of course, here too an agent's attitude may change from 'full belief' to 'suspended judgment' by altering the parameters in the model to place the proposition $L$ outside of the pocket of ordinary full belief, and this likewise may occur without altering the character of the evidence.

So far we've seen that the character of evidence and belief need not match. Imprecise and weak evidence can warrant full belief (Ridgeon), whereas high-thresholded evidence sometimes warrants full belief (table legs) and sometimes does not (lottery tickets). But there is one last point. On Sturgeon's telling, Lockean belief is a form of 'attitudinal imprecision'. So, while the principle of character matching fails to explain the relationship between evidence and Lockean belief, one might think that Sturgeon is at least right in claiming that Lockean belief is imprecise in character. But, alas even this isn't necessarily so, for we may normalize the ratio of risk-to-reward by defining 
$\beta=w / r+w$ along with a classical probability function for some agent $Y, p_{Y}$, over a propositional language. Then, if $Y$ fully $(r: w)$ believes $\varphi$, there is a $\beta \in[0,1]$ such that the agent fully $\beta / 1-\beta$ believes $\varphi$ and $p_{Y}(\varphi)>\beta$ (Kyburg 1990). So, to illustrate, if $Y$ fully $(1: 20)$ believes $\varphi$, then $p_{Y}(\varphi)>20 / 21 \approx 0.95$.

Here then is Character Matching turned inside out. For implicit in the Lockean model for full acceptance is a conjugacy relation that defines full rejection in terms of full acceptance: given some threshold parameter $1 / 2<\theta<1$, a Lockean agent fully accepts $\varphi$ when his credence that $\varphi$ is above $\theta$ just in case the agent fully rejects $\neg \varphi$ when his credence for $\neg \varphi$ is below $1-\theta$. Thus, full Lockean belief and full Lockean disbelief occur in the tails of the 0,1 interval. The pocket of belief, in normalized form, yields a numerically precise credal probability - if it yields one at all—that is within the Lockean pockets of full belief and full disbelief, and it will not yield a numerically precise measurement outside of them. So, for a $(1: 20)$ pocket of full belief, full belief can occur only when credences are between 0.95 and 1 , and full disbelief occurs only when credences are between 0 and 0.05 . It is not necessary that there be numerically precise credences: a qualitative, comparative judgment might be enough to classify all acts in a context as inside the pocket. But, if there are sharp credal probabilities worth considering, you'll find them in Locke's pocket.

ACKNOWLEDGEMENTS This work was supported by award LogiCCC/0001/2007 from the European Science Foundation. A version of this paper was presented at the 2010 APA Pacific Division Meeting in San Francisco. Thanks to Franck Lihoreau, Jonathan Weisberg and Sarah Wright for their comments.

\section{REFERENCES}

Braithwaite, R. B. (1946). Belief and action. Proceedings of the Aristotelian Society 20,1-19.

Hawthorne, J. P. (2004). Knowledge and Lotteries. Oxford: Clarendon Press.

Kyburg, Jr., H. E. (1961). Probability and the Logic of Rational Belief. Middletown, CT: Wesleyan University Press.

Kyburg, Jr., H. E. (1990). Science and Reason. New York: Oxford University Press.

Levi, I. (1980). The Enterprise of Knowledge. Cambridge, MA: MIT Press.

Makinson, D. C. (1965). The paradox of the preface. Analysis 25, 205-207.

Makinson, D. C. (2005). Bridges from Classical to Nonmonotonic Logic. London: King's College Publications.

Paris, J. and R. Simmonds (2009). O is not enough. The Review of Symbolic Logic 2, 298-309.

Sturgeon, S. (2008). Reason and the grain of belief. Noûs 42(1), 139-65.

Wheeler, G. (2006). Rational acceptance and conjunctive/disjunctive absorption. Journal of Logic, Language and Information 15(1-2):49-63. 